\title{
BMJ Open Implementation strategies for interventions to improve the management of chronic kidney disease (CKD) by primary care clinicians: protocol for a systematic review
}

Celia C Kamath, ${ }^{\oplus 1}$ Claudia C Dobler, ${ }^{\circ}$ Michelle A Lampman, ${ }^{1}$ Patricia J Erwin, ${ }^{3}$ John Matulis, ${ }^{4}$ Muhamad Elrashidi, ${ }^{4}$ Rozalina Grubina McCoy, ${ }^{\circ}$ Mouaz Alsawaz, ${ }^{2}$ Atieh Pajouhi, ${ }^{4}$ Amrit Vasdev, ${ }^{4}$ Nilay D Shah, ${ }^{1}$ M Hassan Murad, ${ }^{2}$ Bjorg Thorsteinsdottir ${ }^{4}$

To cite: Kamath CC, Dobler CC, Lampman MA, et al. Implementation strategies for interventions to improve the management of chronic kidney disease (CKD) by primary care clinicians: protocol for a systematic review. BMJ Open 2019;9:e027206. doi:10.1136/ bmjopen-2018-027206

- Prepublication history and additional material for this paper are available online. To view these files, please visit the journal online (http://dx.doi. org/10.1136bmjopen-2018027206).

Received 11 0ctober 2018 Revised 30 May 2019 Accepted 03 July 2019
Check for updates

(C) Author(s) (or their employer(s)) 2019. Re-use permitted under CC BY-NC. No commercial re-use. See rights and permissions. Published by BMJ.

For numbered affiliations see end of article.

Correspondence to

Dr Celia C Kamath;

kamath.celia@mayo.edu

\section{ABSTRACT}

Introduction There is a considerable implementation gap in managing early stage chronic kidney disease (CKD) in primary care despite the high prevalence and risk for increased morbidity and mortality associated with CKD. This systematic review aims to synthesise the evidence of efficacy of implementation interventions aimed at primary care practitioners (PCPs) to improve CKD identification and management. We further aim to describe the interventions' behavioural change components.

Methods and analysis We will conduct a systematic review of studies from 2000 to October 2017 that evaluate implementation interventions targeting PCPs and which include at least one clinically meaningful CKD outcome. We will search several electronic data bases and conduct reference mining of related systematic reviews and publications. An interdisciplinary team will independently and in duplicate, screen publications, extract data and assess the risk of bias. Clinical outcomes will include all clinically meaningful medical management outcomes relevant to CKD management in primary care such as blood pressure, chronic heart disease and diabetes target achievements. Quantitative evidence synthesis will be performed, where possible. Planned subgroup analyses include by (1) study design, (2) length of follow-up, (3) type of intervention, (4) type of implementation strategy, (5) whether a behavioural or implementation theory was used to guide study, (6) baseline CKD severity, (7) patient minority status, (8) study location and (9) academic setting or not.

Ethics and dissemination Approval by research ethics board is not required since the review will only include published and publicly accessible data. Review findings will inform a future trial of an intervention to promote uptake of CKD diagnosis and treatment guidelines in our primary care setting and the development of complementary tools to support its successful adoption and implementation. We will publish our findings in a peerreviewed journal and develop accessible summaries of the results.

PROSPERO registration number CRD42018102441.

\section{Strengths and limitations of this study}

- This protocol conforms to the Preferred Reporting for Systematic review and Meta-analysis Protocols 2015 statement guidelines.

- The planned evidence synthesis will shed light on what works to influence primary care practitioners (PCPs) to adopt guidelines for early detection and management of chronic kidney disease (CKD) in primary care.

- The focus on use and reporting of implementation strategies will highlight the behavioural change strategies likely to enhance effectiveness of interventions.

- The evidence synthesis of impact on a range of clinical outcomes in CKD includes the important dimensions of quality of care in CKD.

- The anticipated heterogeneity in reporting and measures of clinical outcomes and interventions may hinder meta-analysis.

\section{BACKGROUND}

Chronic kidney disease (CKD), with a worldwide prevalence rate of $8 \%-16 \%,{ }^{1}$ is considered a considerable public health issue and a risk factor for increased morbidity and mortality. The estimated prevalence of CKD in individuals 60 years and older increases to $25 \%$. CKD often remains undiagnosed or poorly managed in these individuals ${ }^{1-4}$ in spite of existing guidelines for diagnosing and managing the disease. ${ }^{5-8}$ Given the magnitude of the problem and the potential to modify the course of the disease if diagnosed early, the importance of CKD recognition and proactive early management cannot be overstated. Since patients with CKD are often not referred to a nephrologist until late in the 
course of the disease, it is important to optimise management of CKD in primary care. This need is augmented by the relative shortage of nephrologists. ${ }^{9}$ CKD awareness, quality of care and implementation of guidelines have been found to be inadequate among primary care practitioners (PCPs), including underuse of recommended nephroprotective medications in CKD such as ACE inhibitors or angiotensin receptor blockers, inadequate blood pressure control and late nephrology referrals. ${ }^{10-12}$ This review aims to evaluate the evidence for interventions to improve management of CKD in primary care, targeting PCPs.

The evidence to practice divide has been the focus of enquiry, recognising that successful implementation of any practice guidelines is a multifaceted process, involving healthcare professionals' beliefs, knowledge, confidence and commitment, organisation of care processes and other system level factors. ${ }^{9}{ }^{13}$ Qualitative research has identified specific challenges surrounding management of CKD in primary care. ${ }^{14}$ These include issues regarding whether deterioration of renal function represents normal ageing or a genuine disease in elderly people, challenges in achieving blood pressure targets and difficulty explaining the disease to patients without causing anxiety. ${ }^{14}$ Several interventions have been developed to improve the quality of primary care management of CKD. These include reminders, some embedded in electronic medical records, creation of registries, chronic disease management, educational and other continuous quality improvement methods, with a small percentage of these accompanied by behavioural change interventions.

Systematic reviews on interventions targeted at clinicians managing patients with CKD have identified a number of interventions including chronic disease management strategies, ${ }^{15}$ continuous improvement interventions, ${ }^{16}$ e-alerts, ${ }^{17} 18$ pharmacy-facing interventions ${ }^{19}$ and nurse-led disease management programme or models of care interventions for chronic disease. ${ }^{1720}$ Other reviews assessed multifaceted care approaches ${ }^{18}$ and clinical pathways for primary care. ${ }^{21}$ These reviews are however, either not confined to primary care settings or do not include clinically meaningful outcomes. The two reviews that do, ${ }^{15} 22$ do not separate interventions aimed at clinicians from those that are aimed at patients. Thus, there is a need to review interventions that aim to influence clinician behaviour in managing CKD in primary care using clinically relevant medical management markers. We are also interested in the range of interventions, quality of reporting on intervention details, their underlying behavioural rationale and other relevant information that could guide the development of implementation interventions that target the right behaviours to advance the systematic adoption and sustaining of evidence-based CKD management in routine practice.

The objectives of this systematic review are:

1. Synthesise the evidence of the effect of interventions, aimed at PCPs, to improve the detection and management of CKD on clinically relevant medical management outcomes.

2. Map the type of interventions and implementation methods used to detect and manage CKD in primary care.

3. Identify the most successful implementation approach to effect practice change around CKD management in primary care.

\section{METHODS}

This protocol adheres to the Preferred Reporting Items for Systematic Review and Meta-analysis Protocols ${ }^{23}$ (see online supplementary file 1). Considering that the studied interventions are complex (multicomponent), we will follow frameworks suggested for evidence synthesis of complex interventions. ${ }^{24}{ }^{25}$ Therefore, we will focus on determining the characteristics and circumstances in which the interventions prove to be effective rather than focusing on a simple question of efficacy.

\section{Patient and public involvement}

No patients or the public will be involved since this is a systematic review of interventions targeting clinicians. Instead, clinicians will be actively involved in the conceptualisation, literature search, data abstraction, analysis, interpretation and publishing of findings.

\section{Criteria for considering studies for the review \\ Type of studies}

We will include randomised trials (including cluster randomised trials), non-randomised trials, cross-over trials, quasi-randomised trials, before and after studies with a comparator group and cohort studies.

\section{Types of participants}

Studies that evaluated interventions aimed at any healthcare professional practising in the primary care environment, managing care for adult patients aged 18 years or above with CKD or at risk of developing CKD will be included. Studies including adolescents and children as patients will be excluded.

\section{Types of interventions and comparators}

Any intervention where implementation science methods were an integral part or a component of an intervention directed at PCPs and conducted within a primary care setting to enable managing care for patients with CKD or risk of developing CKD will be included. This broad categorisation includes different modalities of intervention delivery. Comparators will include usual care or any other intervention intended to manage care of patients with CKD in a primary care setting, including historical controls.

\section{Types of outcomes}

We required at least one clinically meaningful medical marker or outcome to be assessed in each study. Those could be either process of care measures (eg, proportion 
of patient taking ACE inhibitors), a relevant surrogate (eg, blood pressure or diabetes control) or a hard clinical outcome (eg, mortality, dialysis).

\section{Search methods for identification of studies}

We will search several electronic data bases including PubMed, Elton B. Stephens Co (EBSCO), Cumulative Index to Nursing and Allied Health Literature (CINAHL), Scopus, Ovid Medline, Ovid Cochrane Library, Ovid Embase, Ovid PsycINFO, Ovid Embase and Web of Science. Non-English language manuscripts will be excluded. We will use the Institute of Medicine ${ }^{26}$ recommendation to guide our search strategy (see online supplementary file 2 for sample search strategy). Due to the relatively young field of implementation science, we will focus our attention to published reports from 2000 to October 2017. Controlled vocabulary supplemented with keywords will be used to search the literature. As implementation interventions that promote the adoption and integration of evidence-based practices, interventions and policies are closely related to the fields of quality improvement, improvement science and similar mechanisms of improvement, we will include search terms associated with these fields. Reference mining of relevant publications will be conducted. We will also hand search all systematic reviews on implementation interventions to improve CKD management in primary care. In addition, we will include study protocols of potentially eligible trials at this stage and follow-up to see if these trials had been published by the time of our analysis. The searches described above will be done with the help of an experienced librarian with several years of experience in systematic review searches.

\section{Selection}

We will upload search results into an EndNote V.8 library. To prepare for selecting and abstracting data, reviewers will undergo education to ensure an understanding of the purpose of the review and a background of the field. Understanding of the inclusion and exclusion criteria will also be assessed through testing on a small number of studies. In the first round of screening, two reviewers will consider the potential eligibility of studies identified by the search strategy based on the abstract and title. Reviewers will request the full text versions of all potentially eligible studies. Studies with reviewer disagreement about eligibility based on abstract and title will also undergo full text review. Eligibility at both the abstract and full text level will be assessed in duplicate and independently. Any disagreements will be resolved by consensus; in the absence of consensus, a third reviewer will arbitrate.

\section{Data extraction}

Extraction of data for this study will include characteristics of study participants, details of interventions, the control interventions, contextual factors, outcome measures for effectiveness (separated into primary and secondary) and

\section{Box 1 List 1}

Patient identification: (1) patients identified/registered with CKD, (2) prevalence of CKD, (3) referral to nephrologist, (4) other (state).

Disease progression: (1) change in glomerular filtration rate (GFR), (2) change in proteinuria, (3) other (state).

Lab monitoring (within the last year): (1) Creatinine or GFR, (2) urineprotein/albumin, (3) haemoglobin, (4) other (state).

Medical management: (1) ACE/angiotensin receptor blocker use, (2) hypertensives-number of classes, (3) avoidance of nephrotoxic drugs, (4) any type of dosing inadequacy.

Blood pressure (BP) management. (1) change in $\mathrm{BP},(2)$ achievement of BP ( $\leq 140 / 90)$, (3) achievement of BP ( $\leq 130 / 80$, (4) other (state).

Diabetes management: (1) haemoglobin A1c (HbA1c), (2) achievement of $\mathrm{HbA1C}<7$, (3) other (state).

Cholesterol management. (1) total cholesterol, (2) low-density lipoprotein, (3) high-density lipoprotein, (4) triglycerides, (5) other (state).

harm, measurement instruments used and factors associated with study quality. Discrepancies in data will be adjudicated by consensus. A third reviewer will arbitrate in the absence of consensus.

A preliminary review of the literature indicated considerable heterogeneity of the types and measurement methods of clinical outcomes in potentially eligible studies. We also observed a wide variation in intervention and implementation strategies. Given this complexity, we included primary care clinicians, health services researchers with implementation science experience and a systematic review methodologist in the team conducting this evidence synthesis. The team met twice to refine the scope of the review, define the specific questions we sought to answer and develop consensus on definitions of interventions and outcomes. Informed by the panel discussions, we created a list of clinically relevant medical management markers for CKD prevention and management to guide the prioritisation of abstraction for clinical variables (see list 1 in box 1 ).

We will create and pilot a standardised data abstraction sheet using Excel software with options to include manual entry and dropdown menus. The latter option for clinical outcomes, for example, will enable abstractors to choose the relevant clinical outcomes and their respective measures with ease.

Implementation interventions will be broadly categorised using elements of the Chronic Care model ${ }^{27}{ }^{28}$ and further detailed in terms of implementation strategies used, using the Expert Recommendations for Implementing Change (ERIC) framework. ${ }^{29} 30$ This is a checklist that details 73 different strategies for implementation. The broad categorisations of implementation strategies are included in list 2 (see list 2 in box 2). More granular details of the intervention strategies under each category can be found in Waltz et al..$^{30}$ Clinicians will abstract clinical data, while two implementation science researchers will dissect and abstract details on the interventions, implementation strategies and delivery modalities. The 


\section{Box 2 List 2}

1. Use evaluative and iterative strategies.

2. Provide interactive assistance.

3. Adapt and tailor to context.

4. Develop stakeholder interrelationships.

5. Train and educate stakeholders.

6. Support clinicians.

7. Encourage consumers.

8. Use financial strategies.

9. Change infrastructure.

lead author will coordinate integrations of these two separate abstraction efforts.

Relevant details of each study context will be abstracted, using guidance from the Consolidated Framework Implementation Research model ${ }^{31}$ described and noted, and used in the narrative analysis. We will also abstract other contextual variables, such as country and academic medical setting versus non-academic settings. Authors of the primary studies will be contacted for clarification if data included in the publication is missing, unclear or in a format that is difficult to extract. Author contact will be initiated by email to the corresponding author. If the email is unavailable, we will search the Internet to find a current email address. If the first author is not the corresponding author, the first author will be carbon copied on all emails to the corresponding author if their email is available. Authors will be given a week to respond to emails at which time a follow-up email will be sent. If no response is received for yet another 2 weeks, we will attempt to contact the author by telephone. If this was not possible, the authors will be classified as not contactable.

\section{Methodological quality and certainty in the evidence}

We will use the Cochrane Collaboration's risk of bias tool $^{32}$ to evaluate the methodological quality of included studies. Reviewers will assess the adequacy of randomisation sequence generation, concealment of treatment arm allocation, blinding of participants and outcome assessors, the degree and potential impact of missing data, the likelihood of incomplete reporting and the potential role of conflicting interests.

For non-randomised studies, we will adapt the New Castle Ottawa instrument to assess risk of bias, focusing on cohort selection, comparability and outcome ascertainment. ${ }^{33}$ Risk of bias abstraction will be elicited with dropdown menus.

We will also assess the quality of reporting on implementation outcomes, using an adapted Template for Intervention Description and Replication checklist. ${ }^{34}$

The certainty in evidence (confidence in the effect) will be evaluated using adaptations of Grading of Recommendations, Assessment, Development and Evaluation) for complex interventions ${ }^{35}$ and narrative synthesis. ${ }^{36}$

\section{Meta-analysis}

When possible, we will generate meta-analytical estimates of treatment effects. We will use the random-effects model because of anticipated heterogeneity in studies' settings and populations. The clinical outcomes that have at least three or more studies with relevant data will be pooled. For clustered randomised trials, we will calculate 'effective sample sizes' for each intervention group and combine with other randomised controlled trials (RCTs) ${ }^{37}$ Sensitivity analyses will be conducted to evaluate the robustness of the findings. We will use Stata statistical software package to conduct the analyses. ${ }^{38}$ Other clinical outcomes, not amenable to meta-analysis, will be summarised narratively and tabulated in terms of significant findings.

We will map interventions and contextual characteristics of the study with types of implementation strategies used, through tabulation methods, looking for patterns of association between them.

Narrative analysis will be conducted by noting the studies with intervention success. We define intervention success in terms of desired direction and magnitude of effectiveness on key clinical endpoints such as blood pressure control. We will thematically analyse features of these studies in terms of components of interventions, their associated implementation strategies and contextual features to identify factors associated with success.

\section{Subgroups}

Subgroup analyses will be conducted to explore the causes of inconsistency and different effects in subgroups. We plan to conduct the following a priori defined subgroup analyses: (1) study design (RCT or cohort design), (2) length of follow-up (12 months, 12-24 months, over 24 months), (3) type of intervention (guideline based alerts, shared care, interventions aimed at pharmacists, collaborative and tailored interventions, (4) type of implementation (based on ERIC classification), (5) whether or not a behavioural or implementation theory was used to guide the study, (6) baseline CKD severity, (7) patient minority status, (8) study location (UK, other European countries, Australia, Canada, USA and other) and (9) academic setting or not. If the intervention structure proves highly variable and we have a sufficient number of studies, we may attempt to explain variation in effects by conducting a metaregression analysis of intervention and implementation characteristics by clinical outcome.

\section{Missing data and sensitivity analyses}

We will attempt to contact authors for missing data. In the event that the data are still unavailable, we will use a complete case analysis and conduct sensitivity analysis using methods described by Ebrahim $e t a l^{39}$ for continuous variables and $\mathrm{Akl} e t a l^{40}$ for dichotomous variables.

\section{Publication bias}

If the number of studies per analysis is over 10 , we will assess publication bias by using funnel plots, plotting the 
estimate of effect of trials by the inverse of its SE. A significant publication bias will be suspected if, using Egger's test, ${ }^{41}$ the $p$ value is $<0.10$. If funnel plots are not possible, we will look at trial registries and unpublished data to assess potential publication bias.

\section{DISCUSSION}

CKD detection and management in primary care is a challenging task, given the competing demands on clinicians and the asymptomatic nature of the disease. Yet, its importance cannot be emphasised enough given the upstream health and cost implications of disease progression in patients at risk for CKD. Several disparate interventions have been tried over the last couple of decades targeting PCPs as well as patients. This review focuses on interventions aimed at PCPs, evaluating what works, and deconstructing the nature of the interventions and their implementation to provide guidance as to what works under what circumstances in preparation for an intervention study in primary care.

This study may encounter several limitations including a high degree of heterogeneity in the interventions as well as the heterogeneity of clinical outcomes in this clinical area. Pooling data from heterogeneous populations and interventions carries inherent uncertainty. We acknowledge that the studies will include participants with considerable variation in baseline risk. We also acknowledge the possibility that non-randomised participants in the control arm might have different baseline risks than participants in the intervention arm. We chose to exclude studies including solely interventions aimed at educating or informing patients even if they met all other inclusion criteria for this study as we are primarily interested in modifying clinician behaviour towards increased quality of care. Our systematic review will identify evidence gaps and provide information on which interventions targeted at clinicians work best to improve the care of patients at risk of CKD or with an established diagnosis of CKD in primary care.

\section{Ethics and dissemination}

Approval by research ethics board is not required since the review will only include published and publicly accessible data. Review findings will inform a future trial of an intervention to promote uptake of CKD diagnosis and treatment guidelines in our primary care setting and the development of complementary tools to support its successful adoption and implementation. We will publish our findings in a peer-reviewed journal and develop accessible summaries of the results.

\section{Author affiliations}

${ }^{1}$ Health Care Policy and Research, Robert D and Patricia E Kern Center for the Science of Health Care Delivery, Mayo Clinic, Rochester, Minnesota, USA ${ }^{2}$ Evidence-Based Practice Center, Robert D and Patricia E Kern Center for the Science of Health Care Delivery, Mayo Clinic, Rochester, Minnesota, USA ${ }^{3}$ Mayo Medical Libraries, Mayo Clinic College of Medicine, Rochester, Minnesota, USA
${ }^{4}$ Division of Community Internal Medicine, Mayo Clinic, Rochester, Minnesota, USA ${ }^{5}$ Division of Endocrinology, Department of Medicine, Mayo Clinic, Rochester, Minnesota, USA

Contributors CCK and BT are the guarantors of the review. CCK conceptualised, designed and coordinated the study and created the initial draft and final manuscript. BT contributed to conceptualisation and design of the study, helped revise the manuscript and provided final approval. CCD and MAL helped in the design of the study, helped revise the manuscript and provided final approval. NDS and MHM provided guidance in conceptualising and designing the study, revised the final draft and provided final approval. PJE was instrumental in the literature search strategy, helped with design of the study and provided final approval. JM, ME, RGM, MA, AP and AV helped in various stages of conceptualising and designing the study, contributed towards the revision of the manuscript and provided final approval.

Funding The authors have not declared a specific grant for this research from any funding agency in the public, commercial or not-for-profit sectors.

Competing interests None declared.

Patient consent for publication Not required.

Provenance and peer review Not commissioned; externally peer reviewed.

Open access This is an open access article distributed in accordance with the Creative Commons Attribution Non Commercial (CC BY-NC 4.0) license, which permits others to distribute, remix, adapt, build upon this work non-commercially, and license their derivative works on different terms, provided the original work is properly cited, appropriate credit is given, any changes made indicated, and the use is non-commercial. See: http://creativecommons.org/licenses/by-nc/4.0/.

\section{REFERENCES}

1. Allen AS, Forman JP, Orav EJ, et al. Primary care management of chronic kidney disease. J Gen Intern Med 2011;26:386-92.

2. Collins AJ, Foley RN, Chavers B, et al. Us renal data system 2013 annual data report. Am J Kidney Dis 2014;63(1 Suppl).

3. Coresh J, Selvin E, Stevens LA, et al. Prevalence of chronic kidney disease in the United States. JAMA 2007;298:2038-47.

4. Jha V, Garcia-Garcia G, Iseki K, et al. Chronic kidney disease: global dimension and perspectives. Lancet 2013;382:260-72.

5. Grol R, Grimshaw J. From best evidence to best practice: effective implementation of change in patients' care. Lancet 2003;362:1225-30.

6. Levey AS, Schoolwerth AC, Burrows NR, et al. Comprehensive public health strategies for preventing the development, progression, and complications of CKD: report of an expert panel convened by the centers for disease control and prevention. Am J Kidney Dis 2009;53:522-35.

7. National Kidney Foundation. K/DOQI clinical practice guidelines for chronic kidney disease: evaluation, classification, and stratification. Am J Kidney Dis 2002;39(2 Suppl 1):S1-266.

8. Levey AS, Coresh J, Balk E, et al. National kidney Foundation practice guidelines for chronic kidney disease: evaluation, classification, and stratification. Ann Intern Med 2003;139:137-47.

9. Field M. Addressing the global shortage of nephrologists. Nat Clin Pract Nephrol 2008;4:583.

10. Boulware LE, Troll MU, Jaar BG, et al. Identification and referral of patients with progressive CKD: a national study. Am J Kidney Dis 2006;48:192-204.

11. Philipneri MD, Rocca Rey LA, Schnitzler MA, et al. Delivery patterns of recommended chronic kidney disease care in clinical practice: administrative claims-based analysis and systematic literature review. Clin Exp Nephrol 2008;12:41-52.

12. Chan MR, Dall AT, Fletcher KE, et al. Outcomes in patients with chronic kidney disease referred late to nephrologists: a metaanalysis. Am J Med 2007;120:1063-70.

13. Suki WN. Are physicians assistants the answer to a shortage of nephrologists? Am J Kidney Dis 1999;33:796-7.

14. Richards N, Harris K, Whitfield M, et al. The impact of populationbased identification of chronic kidney disease using estimated glomerular filtration rate (eGFR) reporting. Nephrol Dial Transplant 2008;23:556-61.

15. Galbraith L, Jacobs C, Hemmelgarn BR, et al. Chronic disease management interventions for people with chronic kidney disease in primary care: a systematic review and meta-analysis. Nephrol Dial Transplant 2018;33:112-21. 
16. Silver SA, Bell CM, Chertow GM, et al. Effectiveness of quality improvement strategies for the management of CKD: a metaanalysis. Clin J Am Soc Nephrol 2017;12:1601-14.

17. Xu H, Mou L, Cai Z. A nurse-coordinated model of care versus usual care for chronic kidney disease: meta-analysis. J Clin Nurs 2017;26:1639-49.

18. Bello AK, Qarni B, Samimi A, et al. Effectiveness of multifaceted care approach on adverse clinical outcomes in nondiabetic CKD: a systematic review and meta-analysis. Kidney Int Rep 2017;2:617-25

19. Tesfaye WH, Castelino RL, Wimmer BC, et al. Inappropriate prescribing in chronic kidney disease: a systematic review of prevalence, associated clinical outcomes and impact of interventions. Int J Clin Pract 2017;71. doi:10.1111/ijcp.12960. [Epub ahead of print: 23 May 2017].

20. Chen C-C, Chen Y, Liu X, et al. The efficacy of a nurse-led disease management program in improving the quality of life for patients with chronic kidney disease: a meta-analysis. PLoS One 2016;11:e0155890.

21. Elliott MJ, Gil S, Hemmelgarn BR, et al. A scoping review of adult chronic kidney disease clinical pathways for primary care. Nephrol Dial Transplant 2017;32:838-46.

22. Tsang JY, Blakeman T, Hegarty J, et al. Understanding the implementation of interventions to improve the management of chronic kidney disease in primary care: a rapid realist review. Implement Sci 2016;11.

23. Shamseer L, Moher D, Clarke M, et al. Preferred reporting items for systematic review and meta-analysis protocols (PRISMA-P) 2015: elaboration and explanation. BMJ 2015;350:g7647.

24. Viswanathan M, McPheeters ML, Murad MH, et al. AHRQ series on complex intervention systematic reviews-paper 4: selecting analytic approaches. J Clin Epidemiol 2017;90:28-36.

25. Pigott T, Noyes J, Umscheid CA, et al. AHRQ series on complex intervention systematic reviews-paper 5: advanced analytic methods. $J$ Clin Epidemiol 2017:90:37-42.

26. Eden J, Levit L, Berg A, et al. Institute of Medicine Committee on Standards for Systematic Reviews of Comparative Effectiveness R. In: Finding what works in health care: standards for systematic reviews, 2011.

27. Bodenheimer T, Wagner EH, Grumbach K. Improving primary care for patients with chronic illness: the chronic care model, part 2. JAMA 2002;288:1909-14.
28. Bodenheimer T, Wagner EH, Grumbach K. Improving primary care for patients with chronic illness. JAMA 2002;288:1775-9.

29. Powell BJ, Waltz TJ, Chinman MJ, et al. A refined compilation of implementation strategies: results from the expert recommendations for implementing change (ERIC) project. Implement Sci 2015;10.

30. Waltz TJ, Powell BJ, Matthieu MM, et al. Use of concept mapping to characterize relationships among implementation strategies and assess their feasibility and importance: results from the expert recommendations for implementing change (ERIC) study. Implement Sci 2015;10.

31. Damschroder LJ, Aron DC, Keith RE, et al. Fostering implementation of health services research findings into practice: a consolidated framework for advancing implementation science. Implement Sci 2009;4.

32. Higgins JPT, Altman DG, Gøtzsche PC, et al. The Cochrane collaboration's tool for assessing risk of bias in randomised trials. BMJ 2011;343:d5928.

33. Wells G, Shea B, O'Connell D, et al. Pages on July 2012;15.

34. Hoffmann TC, Glasziou PP, Boutron I, et al. [Better Reporting of Interventions: Template for Intervention Description and Replication (TIDieR) Checklist and Guide]. Gesundheitswesen 2016;78:175-88

35. Murad $\mathrm{MH}$, Almasri J, Alsawas M, et al. Grading the quality of evidence in complex interventions: a guide for evidence-based practitioners. Evid Based Med 2017;22:20-2.

36. Murad MH, Mustafa RA, Schünemann $\mathrm{HJ}$, et al. Rating the certainty in evidence in the absence of a single estimate of effect. Evid Based Med 2017;22:85-7.

37. Higgins J, Green S. Cochrane Handbook for Systematic Reviews of Interventions 5.10 [WWW document], 2009. The Cochrane Collaboration

38. LLC SC. Pages. Available: www.stata.com

39. Ebrahim S, Akl EA, Mustafa RA, et al. Addressing continuous data for participants excluded from trial analysis: a guide for systematic reviewers. J Clin Epidemiol 2013;66:1014-21.

40. Akl EA, Johnston BC, Alonso-Coello P, et al. Addressing dichotomous data for participants excluded from trial analysis: a guide for systematic reviewers. PLoS One 2013;8:e57132.

41. Egger M, Davey Smith G, Schneider M, et al. Bias in meta-analysis detected by a simple, graphical test. BMJ 1997;315:629-34. 